\title{
Interim Analysis Schedule
}

National Cancer Institute

\section{Source}

National Cancer Institute. Interim Analysis Schedule. NCI Thesaurus. Code C142583.

Specification of the intervening time period or stage of information when interim analyses are planned to take place. 